\title{
Dayside global ionospheric response to the major interplanetary events of October 29-30, 2003 "Halloween Storms"
}

\author{
A. J. Mannucci, ${ }^{1}$ B. T. Tsurutani, ${ }^{1}$ B. A. Iijima, ${ }^{1}$ A. Komjathy, ${ }^{1}$ A. Saito, ${ }^{2}$ \\ W. D. Gonzalez, ${ }^{3}$ F. L. Guarnieri, ${ }^{3}$ J. U. Kozyra, ${ }^{4}$ and R. Skoug ${ }^{5}$ \\ Received 12 September 2004; accepted 21 March 2005; published 4 May 2005.
}

[1] We demonstrate extreme ionospheric response to the large interplanetary electric fields during the "Halloween" storms that occurred on October 29 and 30, 2003. Within a few $(2-5)$ hours of the time when the enhanced interplanetary electric field impinged on the magnetopause, dayside total electron content increases of $\sim 40 \%$ and $\sim 250 \%$ are observed for the October 29 and 30 events, respectively. During the Oct 30 event, $\sim 900 \%$ increases in electron content above the CHAMP satellite $(\sim 400 \mathrm{~km}$ altitude) were observed at mid-latitudes $( \pm 30$ degrees geomagnetic). The geomagnetic storm-time phenomenon of prompt penetration electric fields is a possible contributing cause of these electron content increases, producing dayside ionospheric uplift combined with equatorial plasma diffusion along magnetic field lines to higher latitudes, creating a "daytime super-fountain" effect. Citation: Mannucci, A. J., B. T. Tsurutani, B. A. Iijima, A. Komjathy, A. Saito, W. D. Gonzalez, F. L. Guarnieri, J. U. Kozyra, and R. Skoug (2005), Dayside global ionospheric response to the major interplanetary events of October 29-30, 2003 "Halloween Storms," Geophys. Res. Lett., 32, L12S02, doi:10.1029/2004GL021467.

\section{Introduction}

[2] The ionospheric response to changes in solar wind conditions has been the subject of concerted study for several decades [Blanc and Richmond, 1980; Prolss, 1997; Buonsanto, 1999; Richmond and Lu, 2000; FullerRowell et al., 1997]. The complex interaction of the solar wind, magnetosphere, thermosphere and ionosphere causes dynamical changes over a variety of spatial and temporal scales. In this paper we report very large hemispheric-scale daytime changes in plasma structure of the Earth's ionosphere during the extreme interplanetary (geomagnetic storm) events of October 29-30, 2003 ("Halloween storms"). A factor contributing to the TEC increases may be daytime eastward-directed electric fields penetrating promptly from high to low latitudes, as has been reported previously but primarily for dusk to midnight local times [Fejer, 2002; Tanaka and Ohtaka, 1996; Vlasov et al., 2003; Fejer and Scherliess, 1997; Greenspan et al., 1991].

\footnotetext{
${ }^{1}$ Jet Propulsion Laboratory, California Institute of Technology, Pasadena, California, USA.

${ }^{2}$ Department of Geophysics, Kyoto University, Kyoto, Japan.

${ }^{3}$ Instituto de Pesquisas Espaciais, São José dos Campos, Brazil.

${ }^{4}$ Department of Atmospheric, Oceanic and Space Sciences, University of Michigan, Ann Arbor, Michigan, USA.

${ }_{5}^{5}$ Los Alamos National Laboratory, Los Alamos, New Mexico, USA.
}

[3] We use Global Positioning System receiver data acquired from ground-based receivers and a low Earthorbiting receiver. Space-borne receivers using upward viewing antennas measure the electron content above the satellite altitude (CHAMP at $400 \mathrm{~km}$ altitude). CHAMP measurements along with DMSP ion drift measurements suggest that large ionospheric uplift occurs due to electric fields.

\section{Observations}

[4] Figure 1 shows data associated with two interplanetary coronal mass ejections (ICMEs) plus their associated shocks that were detected by the ACE spacecraft upstream of the Earth on October 29 and 30, 2003, including proton speed, temperature, density, and magnetic fields from ACE, and Dst index values. The ICMEs causing the magnetic storms were related to X-class solar flares that occurred on October 28 and October 29, 2003 [see Tsurutani et al., 2005].

[5] The first geomagnetic storm of interest is associated with an abrupt Dst decrease at $\sim 1400$ UT on October 29. $B_{z}$ initially has a magnitude of $+8 \mathrm{nT}$ but continues to turn steadily southward, reaching a value of $-30 \mathrm{nT}$ at $1910 \mathrm{UT}$. This $B_{z}$ southward turning, part of a magnetic cloud [Skoug et al., 2004], combined with solar wind velocities in the range $1200-2100 \mathrm{~km} / \mathrm{sec}$, causes a geomagnetic storm and peak Dst excursion of $-350 \mathrm{nT}$ recorded at 0125 UT on October 30. The shock of a second ICME occurs at $\sim 1650$ UT on October 30. The strong southward magnetic field at and just after the shock causes another geomagnetic storm that commences at 1845 UT (during the recovery phase of the previous storm). This last storm is the most intense, with peak Dst of $-390 \mathrm{nT}$ at $2315 \mathrm{UT}$ on October 30.

[6] The main topic of this paper are these $B_{z}$ southward events followed by unusually large daytime ionospheric responses as measured by ionospheric total electron content (TEC) data obtained from Global Positioning System (GPS) receivers. Using techniques mentioned extensively in the literature [Mannucci et al., 1998, 1999] a dualfrequency GPS receiver can measure the total electron content of the ionosphere/plasmasphere system between the receiver and satellite, with high precision (0.01 TECU is typical, 1 TECU $=10^{16} \mathrm{el} / \mathrm{m}^{2}$ ) and reasonable accuracy $(\sim 1-3$ TECU).

[7] Vertical TEC data derived from a global network of $\sim 100$ GPS receivers are plotted in Figure 2 for several days leading up to and through the geomagnetic storm periods of October 29-30, 2003. An obliquity function is used to estimate the vertical TEC from the slant TEC measurements obtained above 10 degrees elevation angle: we assume that 

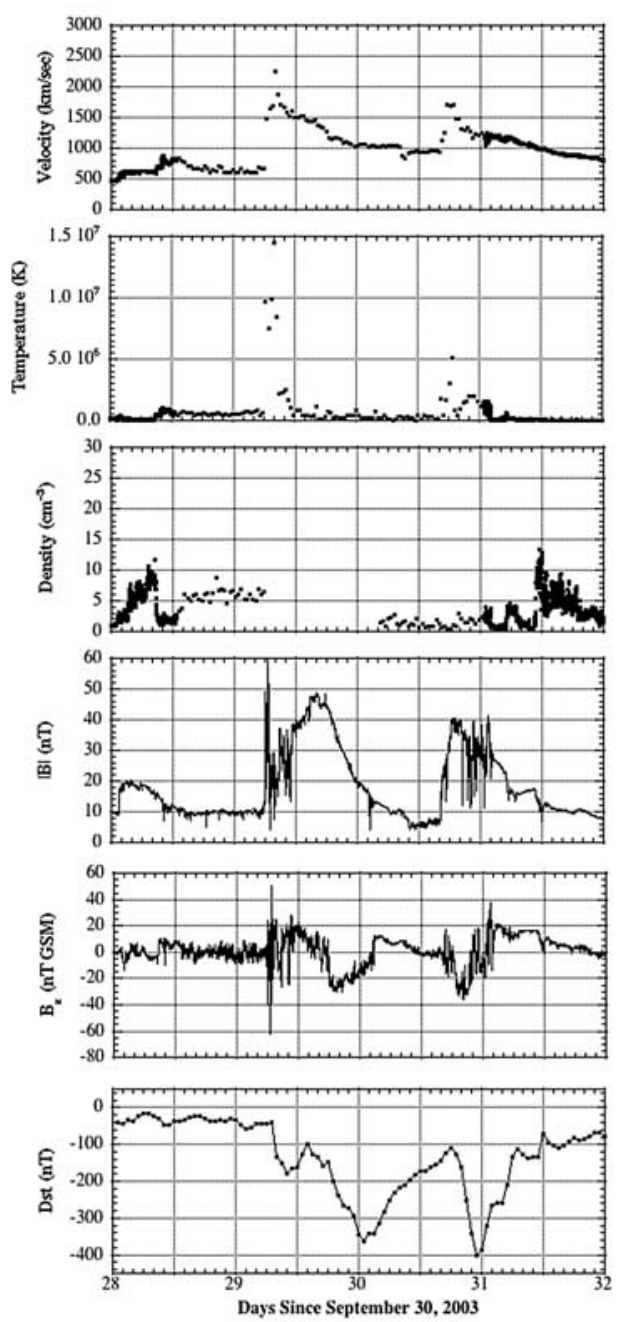

Figure 1. The interplanetary event of late October 2003. The interplanetary data were obtained by the ACE spacecraft, including the proton speed in $\mathrm{km} / \mathrm{sec}$, the proton temperature $(\mathrm{K})$, proton density $\left(\mathrm{cm}^{-3}\right)$, and magnetic field magnitude (nT). The magnetic field component $B_{z}$ is plotted for GSM coordinates. The southward- $B_{z}$ events on October 29 and 30 lead to large geomagnetic storms and ring current increases as measured by the Dst index.

the ionosphere occupies a spherical slab of uniform vertical electron density between 450 and $650 \mathrm{~km}$ altitude. The latitude and longitude at which the ground-to-satellite lineof-sight intersects the ionosphere is computed using a spherical shell at $450 \mathrm{~km}$ altitude.

[8] The TEC measurements in the local time range 1400-1600 LT and magnetic latitude range \pm 40 degrees (IGRF) [Richmond, 1995] were averaged every 30-minutes of Universal Time (UT), to examine TEC behavior at low to middle latitudes. The resultant time series of average vertical TEC versus UT is plotted along with the computed interplanetary electric field (IEF), derived from the product $V_{x} \times B_{z}$ (GSM coordinates) measured by the plasma and magnetic field instruments onboard the ACE spacecraft [Skoug et al., 2004]. The IEF data is offset by the propagation time from ACE to the magnetospheric bow shock and is only plotted for values where the product $V_{x} \times B_{z}$ is positive.
[9] Also plotted in magenta in Figure 2 is the average TEC plus its standard deviation, computed from the scatter of all vertical TEC data contributing to the average value. The observed TEC increases of $\sim 50 \%-250 \%$ (after the larger IEF increase) are much larger than the day-to-day variability observed prior to the IEF increases. The number of points contributing to the averages ranged from 50 to 600 , depending on UT, as the number of sites within the relevant local time varied.

[10] Variability of TEC with time shown in Figure 2 is likely due to two factors: variability of the ionosphere itself, and the fact that the GPS receiver distribution varies with longitude. Even if the ionospheric TEC were not changing, the latitude sampling of the receivers used to compute the average is changing with UT, thereby changing the locations at which TEC is measured. Ionospheric features that exhibit latitudinal structure will change the average as a function of time. Despite these sources of uncertainty, a strong correlation is observed between enhanced TEC and $\mathrm{B}_{\mathrm{z}}$ south (enhanced interplanetary electric field), which is clearly distinguished from the preceding quiet-time data. The local time/latitude range used to compute the average was chosen to emphasize effects within a broad daytime, low latitude region.

[11] Daytime observations of the TEC above the CHAMP satellite altitude of $400 \mathrm{~km}$ on October 30 were available from the upward-viewing GPS antenna, plotted in Figure 3. Slant measurements obtained above 40 degrees elevation are scaled to estimate vertical TEC above the satellite altitude, using a geometric factor derived by assuming the plasma occupies a spherical shell ionosphere of uniform density and $700 \mathrm{~km}$ thickness above the CHAMP altitude. There

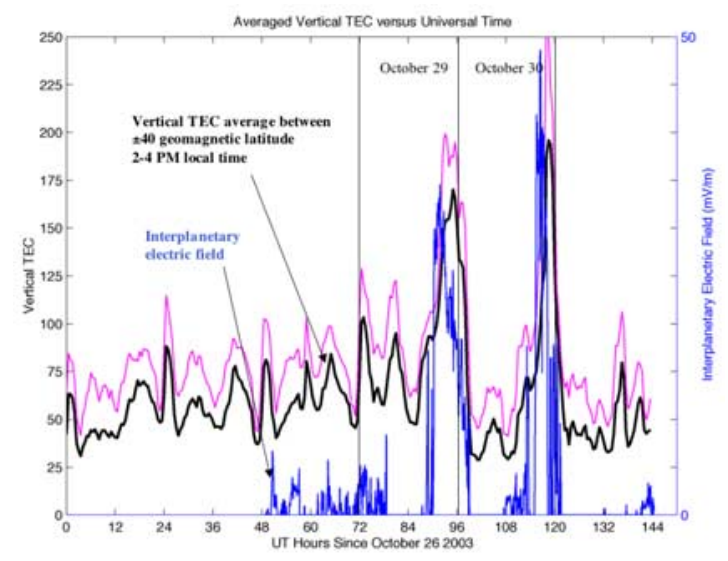

Figure 2. The average of estimated vertical ionospheric total electron content from the ground GPS network is plotted for several days leading up to and including the Halloween 2003 storms. The average (solid black line) is calculated over the range \pm 40 degrees apex latitude, for local times between 14-16 LT. The interplanetary electric field as measured by the ACE spacecraft $\left(v_{x} \times B_{z}\right.$, GSM coordinates) is plotted in blue, delayed according to the velocity measured by ACE (Figure 1), assuming a distance between ACE and the magnetopause of $1.4 \times 10^{6} \mathrm{~km}$. The TEC added to its standard deviation computed for all measurements at a given epoch is also shown (solid magenta line). 


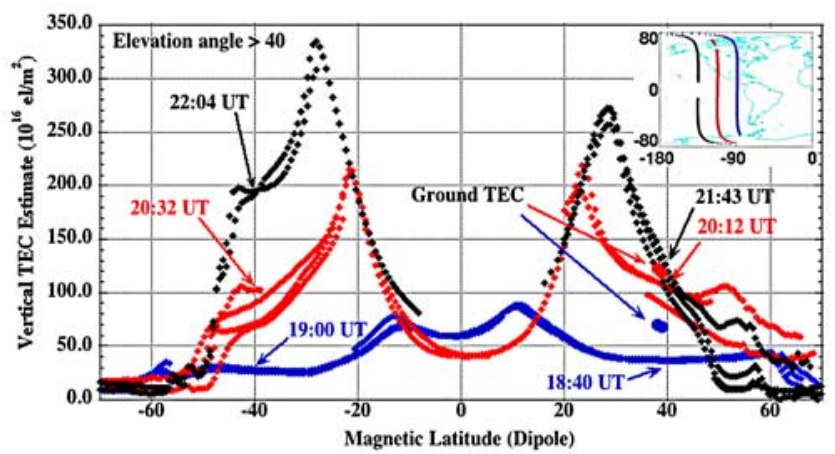

Figure 3. The supersatellite integrated electron content (IEC) as measured by the CHAMP spacecraft is shown just prior (blue trace) and after (red and black traces) the onset of the interplanetary event of October 30 (see Figure 1). The different sets of points for each trace correspond to IEC measured towards the different GPS satellites at each epoch, which are all used to estimate vertical TEC directly above the CHAMP satellite altitude of $400 \mathrm{~km}$, using an elevation angle cut-off of 40 degrees to reduce the error in the vertical IEC estimation procedure. The local time of the CHAMP orbit ranges from 1230-1330 LT for latitudes within \pm 60 degrees. Points missing near the anomaly trough are due to the elevation angle cut-off. The universal times of the -40 and +40 degree latitude cross-over points are shown for each trace. Also shown in the upper right are the geographical locations of the traces. Total electron content averages from ground data near to the CHAMP ground track are shown as round dots.

are generally several points at a given epoch (seen as multiple traces for a single color) because there can be more than one GPS satellite in view of CHAMP at elevations greater than 40 degrees. The separate traces generally agree, except for the red traces between 40 and 60 degrees latitude, which differ because of the azimuth of the raypaths to the GPS satellites: the lower TEC values are associated with a satellite being tracked in the northwest direction versus northeast/east-directed azimuths for the higher TEC values. These traces differ because of horizontal TEC gradients in the vicinity of the satellite.

[12] Three daytime ascending passes are shown in Figure 3 , one pass before the larger interplanetary $B_{z}$ south event of October 30, and two that occurred afterwards. Prior to the interplanetary event (blue markers), the CHAMP latitudinal profile shows the typical "two-peak" structure similar to an undisturbed equatorial anomaly [Hanson and Moffett, 1966].

[13] The first post-onset pass (red markers), starting at 2012 UT, measures a vastly increased TEC above CHAMP, up to 200 TECU, with the peaks farther apart. This trace begins approximately 1.25 hours after the onset of the sustained IMF southward-B $\mathrm{z}_{\mathrm{z}}$ event at 1900 UT. Inspection of ground-based TEC data from North America (not shown) indicate that temporal changes, not longitudinal gradients, are responsible for the differences between the pre-onset and first post-onset pass. The TEC increases still further to $\sim 350$ TECU in the southern hemisphere approximately 1.5 hours later (black markers, second pass after southward $\mathrm{B}_{\mathrm{z}}$ ). The twin peak features previously identified as an equatorial anomaly now appear at mid-latitudes ( \pm 28 degrees). The ionospheric TEC above CHAMP altitudes has increased by an order of magnitude (900\%) at mid-latitudes $\left(-30^{\circ}\right.$ geomagnetic). Part of the difference between this pass and the previous one may be due to longitudinal TEC gradients, which have begun to build up on the North American west coast, according to groundbased TEC (not shown).

[14] We have plotted select vertical TEC from groundbased GPS receivers in North America (red and blue dots at 38 and 39 degrees geomagnetic latitude), for measurements located within \pm 6 minutes and within \pm 3 degrees longitude of the CHAMP ground track location at the latitude shown. Prior to the interplanetary event (blue dots), the integrated electron content above CHAMP comprises a smaller fraction of the TEC compared to after the interplanetary event (red dots), reinforcing the conclusion that plasma uplift has occurred.

\section{Interpretation and Discussion}

[15] Figure 2 suggests a strong correlation between increased IEF and significant dayside increases in TEC (positive ionospheric storm). A large IEF event that occurred in November of 2001 was studied by Tsurutani et al. [2004], where analysis of multiple data sets led to the conclusion that prompt penetration electric fields contributed to the TEC increase and uplift. Another source of stormtime electric fields at equatorial latitudes is from the thermospheric disturbance dynamo [Blanc and Richmond, 1980], but, as discussed by Richmond and Lu [2000], such daytime electric fields are generally of opposite sign to those reported here. Prompt penetration electric fields tend to be eastward during daytime before shielding has built up [Nopper and Carovillano, 1978]. A daytime eastward electric field raises low latitude plasma upward due to $\mathbf{E} \times \mathbf{B}$ drift, so that more plasma resides at higher altitudes where recombination rates decrease, while additional plasma continues to be generated by solar illumination, resulting in a net increase of electrons above $400 \mathrm{~km}$, consistent with the CHAMP data. We refer to this large TEC enhancement as the "daytime super-fountain effect" associated with magnetic storms, described further by Tsurutani et al. [2004]. Tanaka and Ohtaka [1996] describe a dusk "super-fountain" associated with prompt penetration electric fields that applies to that local time range. High-altitude plasma can take hours to recombine, so we cannot infer from our data the duration of the penetrating electric field. That will require detailed modeling.

[16] To further assess our hypothesis that daytime eastward electric fields contribute to the large TEC increases, we used data from the DMSP satellite (F13) at 1745 local time, which shows evidence of enhanced electric fields between geomagnetic latitudes of -10 and 10 degrees. The drift velocity at DMSP altitude $(840 \mathrm{~km})$ is predominantly negative during the days October 25-30 2003, except for three distinct periods when the drift velocity becomes significantly upward $(>70 \mathrm{~m} / \mathrm{sec})$ : October 29 at 0616 UT and at 1951-2313 UT; and during October 30 1941-2118 UT. The times of these large vertical drift velocities correspond to the periods of southward Bz shown in Figure 1. 
[17] The daytime plasma uplift has potentially important ionospheric/atmospheric effects. Following Figure 3 and assuming an average of 200 TECU $\left(2 \times 10^{14}\right.$ Oxygen ions $/ \mathrm{cm}^{2}$ ) over 0.25 of the Earth's surface is elevated to a height of $800 \mathrm{~km}$ in two hours, the rate of work done by the solar wind corresponds to 3.8 Giga-watts (this is only a small fraction of the solar wind ram energy). The total potential energy is $2.7 \times 10^{13}$ Joules.

[18] If the entire ionosphere is uplifted to heights of $800 \mathrm{~km}$ or more, two prominent effects may occur. With the reduction of plasma density at $\sim 250 \mathrm{~km}$ altitude, ionneutral drag will be substantially reduced and will change the neutral wind system at the equatorial region. Secondly the uplifted heavy ions $\left(\mathrm{O}_{2}+, \mathrm{NO}+\right)$ will diffuse down magnetic field lines to midlatitudes leading to global (dayside) mixing.

\section{Conclusions}

[19] The dramatic TEC increase due to plasma uplift during geomagnetic storms is one of the most dramatic consequences of magnetosphere-thermosphere-ionosphere coupling. Transport of plasma poleward, and possibly uplift at mid-latitudes, causes major mid-latitude plasma increases that may contribute to the extreme TEC gradients at midlatitudes that have been reported as part of TEC plumes associated with subauroral electric fields [e.g., Foster et al., 2002]. If prompt penetration electric fields are playing a role, a better understanding of magnetosphere-ionosphere coupling, including the role of shielding, will clarify our understanding of extreme space weather events over a wide range of latitudes.

[20] Acknowledgments. Portions of the research for this paper were performed at the Jet Propulsion Laboratory, California Institute of Technology under contract with NASA. Work at Los Alamos was performed under the auspices of the U. S. Department of Energy with support from the NASA ACE program. We thank the ACE SWEPAM instrument team and the ACE Science Center for providing the ACE data. We gratefully acknowledge the Center for Space Sciences at the University of Texas at Dallas and the US Air Force for making available the DMSP drift velocity data. One of the authors (AJM) wishes to thank the anonymous referees for their useful suggestions.

\section{References}

Blanc, M., and A. D. Richmond (1980), The ionospheric disturbance dynamo, J. Geophys. Res, 85, 1669-1686.

Buonsanto, M. J. (1999), Ionospheric storms-A review, Space Sci. Rev, $88,563-601$.

Fejer, B. G. (2002), Low latitude storm time ionospheric electrodynamics, J. Atmos. Sol. Terr. Phys., 64, 1401-1408.
Fejer, B. G., and L. Scherliess (1997), Empirical models of storm time equatorial zonal electric fields, J. Geophys. Res, 102, 24,047-24,056.

Foster, J. C., P. J. Erickson, A. J. Coster, J. Goldstein, and F. J. Rich (2002), Ionospheric signatures of plasmaspheric tails, Geophys. Res. Lett., 29(13), 1623, doi:10.1029/2002GL015067.

Fuller-Rowell, T. M., M. V. Codrescu, R G. Roble, and A. D. Richmond (1997), How does the thermosphere and ionosphere react to a geomagnetic storm?, in Magnetic Storms, Geophys. Monogr. Ser., vol. 98, edited by B. T. Tsurutani et al., pp. 203-225, AGU, Washington, D. C.

Greenspan, M. E., C. E. Rasmussen, W. J. Burke, and M. A. Abdu (1991), Equatorial density depletions observed at $840 \mathrm{~km}$ during the great magnetic storm of March 1989, J. Geophys. Res., 96, 13,931-13,942.

Hanson, W. B., and R. J. Moffett (1966), Ionization transport effects in equatorial $F$ region, J. Geophys. Res., 71, 5559-5572.

Mannucci, A. J., B. D. Wilson, D. N. Yuan, C. H. Ho, U. J. Lindqwister, and T. F. Runge (1998), A global mapping technique for GPS-derived ionospheric total electron content measurements, Radio Sci., 33, 565582.

Mannucci, A. J., B. A. Iijima, U. J. Lindqwister, X. Pi, L. Sparks, and B. D. Wilson (1999), GPS and ionosphere, in Reviews of Radio Sci., 19961999, edited by W. R. Stone, Oxford Univ. Press, New York.

Nopper, R. W., and R. L. Carovillano (1978), Polar-equatorial coupling during magnetically active periods, Geophys. Res. Lett., 5, 699-702.

Prolss, G. W. (1997), Magnetic storm associated perturbations of the upper atmosphere, in Magnetic Storms, Geophys. Monogr. Ser, vol. 98, edited by B. T. Tsurutani et al., pp. 227-241, AGU, Washington, D. C.

Richmond, A. D. (1995), Ionospheric electrodynamics using magnetic apex coordinates, J. Geomagn. Geoelectr., 47, 191-212.

Richmond, A. D., and G. Lu (2000), Upper-atmospheric effects of magnetic storms: A brief tutorial, J. Atmos. Sol. Terr. Phys., 62, 1115-1127.

Skoug, R. M., J. T. Gosling, J. T. Steinberg, D. J. McComas, C. W. Smith, N. F. Ness, Q. Hu, and L. F. Burlaga (2004), Extremely high speed solar wind: 29-30 October 2003, J. Geophys. Res., 109, A09102, doi:10.1029/ 2004JA010494

Tanaka, T., and K. Ohtaka (1996), Ionospheric disturbances during lowlatitude auroral events and their association with magnetospheric processes, J. Geophys. Res.-Space Physics, 101(A8), 17,151-17,159.

Tsurutani, B. T., et al. (2004), Global dayside ionospheric uplift and enhancement associated with interplanetary electric fields, J. Geophys. Res., 109, A08302, doi:10.1029/2003JA010342.

Tsurutani, B. T., et al. (2005), The October 28, 2003 extreme EUV solar flare and resultant extreme ionospheric effects: Comparison to other Halloween events and the Bastille Day event, Geophys. Res. Lett., 32, L03S06, doi:10.1029/2004GL021502.

Vlasov, M., M. C. Kelley, and H. Kil (2003), Analysis of ground-based and satellite observations of $F$-region behavior during the great magnetic storm of July 15, 2000, J. Atmos. Sol. Terr. Phys., 65, 1223-1234.

W. D. Gonzalez and F. L. Guarnieri, Instituto de Pesquisas Espaciais, 12201-970, São José dos Campos, CP 515, SP, Brazil.

B. A. Iijima, A. Komjathy, A. J. Mannucci, and B. T. Tsurutani, Jet Propulsion Laboratory, California Institute of Technology, 4800 Oak Grove Drive, Pasadena, CA 91109, USA. (tony.mannucci@jpl.nasa.gov)

J. U. Kozyra, Department of Atmospheric, Oceanic and Space Sciences, University of Michigan, Ann Arbor, MI 48109-2143, USA.

A. Saito, Department of Geophysics, Kyoto University, Kyoto 606-8502, Japan.

R. Skoug, Los Alamos National Laboratory, Los Alamos, NM 87545, USA. 